\title{
A short hard X-ray flare from the blazar NRAO 530 observed by INTEGRAL *
}

\author{
L. Foschini ${ }^{1}$, E. Pian ${ }^{2}$, L. Maraschi ${ }^{3}$, C. M. Raiteri ${ }^{4}$, F. Tavecchio ${ }^{3}$, \\ G. Ghisellini ${ }^{3}$, G. Tosti ${ }^{5}$, G. Malaguti ${ }^{1}$, and G. Di Cocco ${ }^{1}$
}

1 INAF/IASF-Bologna, via Gobetti 101, 40129 Bologna, Italy
e-mail: foschini@iasfbo.inaf.it
2 INAF, Osservatorio Astronomico di Trieste, via G.B. Tiepolo 11, 34131, Trieste, Italy
3 INAF, Osservatorio Astronomico di Brera, via Bianchi 46, 23807, Merate, Italy
4 INAF, Osservatorio Astronomico di Torino, via Osservatorio 20, 10025, Pino Torinese, Italy
5 Osservatorio Astronomico, Università di Perugia, via B. Bonfigli, 06126 Perugia, Italy

Received 22 November 2005 / Accepted 5 January 2006

\section{ABSTRACT}

We report about a short flare from the blazar NRAO 530 occurred on 17 February 2004 and detected serendipitously by the IBIS/ISGRI detector on board INTEGRAL. In the $20-40 \mathrm{keV}$ energy range, the source, that is otherwise below the detection limit, is detected at a level of $\approx 2 \times$ $10^{-10} \mathrm{erg} \mathrm{cm}^{-2} \mathrm{~s}^{-1}$ during a time interval of less than $2000 \mathrm{~s}$, which is about a factor 2 above the detection threshold. At other wavelengths, only nearly-simultaneous radio data are available (1 observation at $2 \mathrm{~cm}$ on 11 February 2004), indicating a moderate increase of the polarization. This appears to be the shortest time variability episode ever detected in a high luminosity blazar at hard X-rays, unless the blazar is contaminated by the presence of an unknown unresolved rapidly varying source.

Key words. galaxies: quasars: general - galaxies: quasars: individual: NRAO 530 - X-rays: galaxies

\section{Introduction}

Blazars are, among active galactic nuclei (AGN), the most luminous and most dramatically variable. These extreme properties are likely due to relativistic aberration in a kilo-parsec jet oriented at a small angle with respect to our line of sight, where plasma moving with a Lorentz factor of $\sim 10-20$ causes radiation boosting and time foreshortening (see reviews by Urry \& Padovani 1995; Wagner \& Witzel 1995; Ulrich et al. 1997). In these sources, the maximum power output and the largest variability amplitudes are observed at high-energies, from Xto $\gamma$-rays, therefore blazars are interesting targets for monitoring with satellites like INTEGRAL (Pian et al. 1999). During its first three years of activity INTEGRAL detected two blazars in outburst, with observations triggered by ground telescopes (S5 0716 + 714 Pian et al. 2005; 3C454.3 Foschini et al. 2005; Pian et al. 2006), and one in high state (S5 $0836+710)$, serendipitously detected during the observation of S5 $0716+$ 714 (Pian et al. 2005). Indeed, thanks to the large field-of-view

\footnotetext{
* Based on observations obtained with INTEGRAL, an ESA mission with instruments and science data centre funded by ESA member states (especially the PI countries: Denmark, France, Germany, Italy, Switzerland, Spain), Czech Republic and Poland, and with the participation of Russia and the USA.
}

of the IBIS instrument onboard INTEGRAL, many blazars, possibly in active state, may be detected serendipitously by the satellite during its long pointings. In order to exploit this advantage, we have systematically searched the INTEGRAL public archive for possible detection of flaring blazars. We report here about the interesting detection of a rapid flare apparently associated with the blazar NRAO 530.

NRAO $530(z=0.902)$ is a blazar belonging to the optically violent variable (OVV) quasar subclass, with emission lines in the optical spectrum, particularly $\mathrm{H} \beta$ with a rest-frame equivalent width of $16 \AA$, smaller than usually observed in OVVs (Junkkarinen 1984; cf. also Veron-Cetty \& Veron 2000). It exhibits strong outbursts in radio (Bower et al. 1997), optical (up to 3 mag in 1977, Pollock et al. 1979; Webb et al. 1988), X-ray (HEAO-1, Marscher et al. 1979), and $\gamma$-ray bands (EGRET/CGRO, Mukherjee et al. 1997), with significant emission above $1 \mathrm{GeV}$ (Lamb \& Macomb 1997). The flare detected by the IBIS/ISGRI instrument on board INTEGRAL occurred in February 2004 with a timescale ( $\approx 2000$ s) much shorter than any variability reported before.

In the following, we present in detail the INTEGRAL data analysis and results (Sect. 2), data obtained quasisimultaneously in the radio (Sect. 3) and a discussion of the relevance of the detected flare for blazars. 


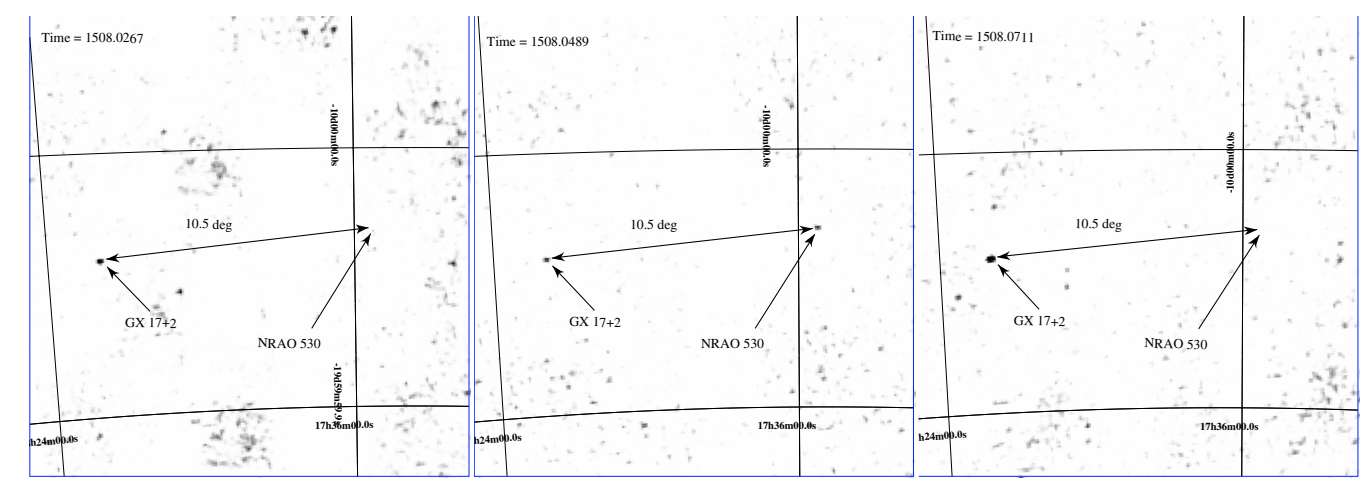

Fig. 1. IBIS/ISGRI significance map in the $20-40 \mathrm{keV}$ energy band for the pointing before, during, and after the detection of NRAO 530.

\section{INTEGRAL data analysis}

The INTEGRAL satellite (Winkler et al. 2003) was launched on 17 October 2002 and it carries two main instruments for the $\gamma$-ray astrophysics, the imager IBIS $(0.02-10 \mathrm{MeV}$, Ubertini et al. 2003) and the spectrometer SPI (0.02-8 MeV, Vedrenne et al. 2003), plus two monitors, JEM-X (3-35 keV, Lund et al. 2003) and OMC ( $V$ filter, Mas-Hesse et al. 2003). Since NRAO 530 is located close to the Galactic Centre $(l=$ $12^{\circ} .0, b=+10.8$ ), a very crowded region, we do not use the data from the spectrometer SPI, since it has an angular resolution of about 2.5 . Also the OMC is strongly limited by the source confusion: its point-spread function (PSF) is 25" (FWHM). The US Naval Observatory Catalog (USNO B1, Monet et al. 2003) reports 12 sources of brightness comparable to that of NRAO 530 inside a circular region of $25^{\prime \prime}$ radius centered around the position of the blazar. Thus, the OMC observations are presumably highly contaminated, and therefore not used.

We also checked the JEM-X data for possible detection, however the source was never optimally located in the monitor field-of-view: in only one of the 11 pointings the source angular distance from the FOV centre was 2.5 , less than the limit of $<3^{\circ}$ required for good JEM-X performance. Using the INTEGRAL pointing $^{1}$ where the source is detected by IBIS (see below), which also corresponds to the minimum distance from the JEM-X FOV center, we determine an upper limit of $30 \mathrm{mCrab}$ in the $5-15 \mathrm{keV}$ energy band ( $5 \sigma$ level, as suggested in Westergaard et al. 2005).

Therefore, in the following we will present only the analysis of the data acquired by IBIS, that is the INTEGRAL instrument with best sensitivity in the hard X-ray range. The analysis has been performed using the Offline Scientific Analysis (OSA) software package ${ }^{2}$, whose algorithms for the reduction and treatment of the IBIS instrument data are described in Goldwurm et al. (2003).

The imager IBIS (Ubertini et al. 2003) is composed by two detectors, ISGRI, sensitive to the radiation between $20 \mathrm{keV}$ and $1 \mathrm{MeV}$ (Lebrun et al. 2003), and PICsIT (175 keV to $10 \mathrm{MeV}$, Di Cocco et al. 2003), both coupled with the same

\footnotetext{
${ }^{1}$ Every single pointing is called "Science Window" $(\mathrm{ScW})$ in the INTEGRAL jargon. A ScW has a duration of about $2000 \mathrm{~s}$.

2 Available at http://isdc.unige.ch/
}

tungsten coded mask. The latter has the pattern of a modified uniformly redundant array (MURA, Gottesman \& Fenimore 1989), with a basic configuration of $53 \times 53$ square pixels $11.2 \mathrm{~mm}$ sized. The mask is separated from the ISGRI layer by about $3200 \mathrm{~mm}$, so that the angular distance of ghosts in the system point spread function (SPSF) of ISGRI is about $10^{\circ} .5$ (Gros et al. 2003). This means that sources with reciprocal angular distances of that size can be confused and specific care is necessary in order to disentangle the different contributions.

On February 17, 2004 (orbit 164), while INTEGRAL was executing the Galactic Centre Deep Exposure (GCDE), ISGRI detected a signal during the time interval between $00^{\mathrm{h}}: 40^{\mathrm{m}}: 05^{\mathrm{s}}$ and $01^{\mathrm{h}}: 09^{\mathrm{m}}: 18^{\mathrm{s}}$ UTC at $\alpha=17^{\mathrm{h}}: 33^{\mathrm{m}}: 04^{\mathrm{s}}$ and $\delta=$ $-13^{\mathrm{h}}: 02^{\mathrm{m}}: 50^{\mathrm{s}}(\mathrm{J} 2000)$. The $3^{\prime}$-radius uncertainty region associated with this centroid is consistent with the blazar NRAO 530 $\left(\alpha=17^{\mathrm{h}} 33^{\mathrm{m}} 02^{\mathrm{s}} .71, \delta=-13^{\circ} 04^{\prime} 49^{\prime} .5, \mathrm{~J} 2000\right)$ and one ghost of the SPSF of the neutron star GX $17+2\left(\alpha=18^{\mathrm{h}} 16^{\mathrm{m}} 01^{\mathrm{s}} .40\right.$, $\delta=-14^{\circ} 02^{\prime} 11^{\prime \prime}(0, \mathrm{~J} 2000)$. The angular distance between the two sources is precisely $10^{\circ} .5$, so that the two SPSF overlap (Fig. 1). However, one can disentangle the contribution of the two sources by analysing their time evolution: if one SPSF is contaminated by the other, both sources should show the same time behaviour.

Since the latest version of OSA 5.0 for IBIS/ISGRI removes the sources located at the potential positions of the ghosts of brightest sources, we used an older version (OSA 3.0), where this feature was not yet implemented. We also cross-checked the consistency of the results obtained with OSA 3.0 with OSA 5.0, however, in the latter case, we adopted an input source catalog containing only NRAO 530 as a source, to by-pass the above protection. Obviously, we also checked that the results on GX $17+2$ were not affected when adopting this solution, by performing a run with OSA 5.0 and the full input catalog.

We compared the behaviour of the background and of the 2 sources by analyzing the 5 pointings before and after the event, corresponding to the time interval between February 16th, 2004 at $21^{\mathrm{h}}: 59^{\mathrm{m}}: 08^{\mathrm{s}}$, and February 17th, 2004 at $03^{\mathrm{h}}: 49^{\mathrm{m}}: 44^{\mathrm{s}}$ UTC. We normalized the count rates of the background and of GX $17+2$ in the 20-40 keV energy band to their weighted averages. For NRAO 530, which has one detection only, we left the count rates unchanged. Figure 2 shows 

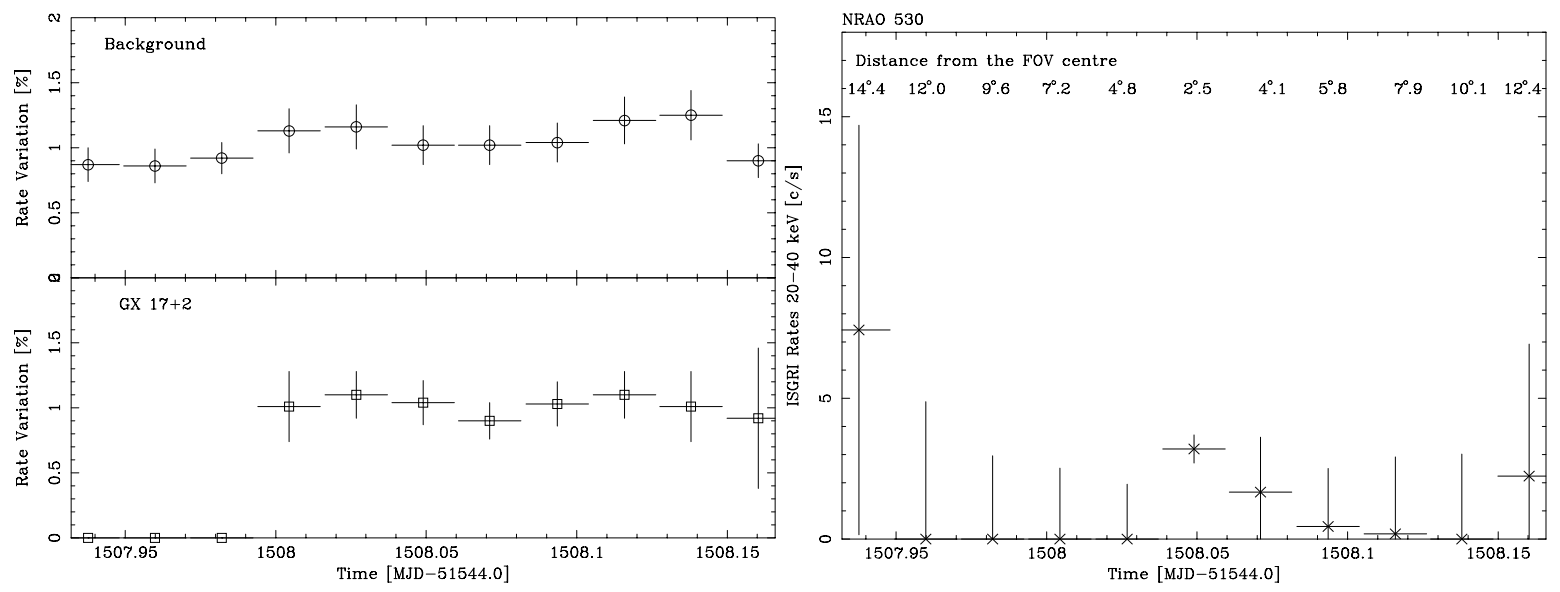

Fig. 2. Left, top: background rates normalized to the weighted average $(79 \pm 12 \mathrm{c} / \mathrm{s})$. Left, bottom: GX $17+2$ rates normalized to the weighted average $(10.9 \pm 0.9 \mathrm{c} / \mathrm{s})$; in the first $3 \mathrm{ScW}$ the source was outside the ISGRI FOV. Right: NRAO 530 rates; upper limits are at $3 \sigma$. In the top of the figure, the distance of the source from the ISGRI FOV centre is shown in every ScW. All the lightcurves are in the 20-40 keV energy band.

the lightcurves of the background, GX $17+2$, and NRAO 530 . While GX 17+2 follows the background time evolution (Fig. 2, left), this is not the case for NRAO 530 (Fig. 2, right). Were the putative detection of NRAO 530 only a ghost of GX $17+2$, then it would follow the same temporal behaviour, which would contradict the derived upper limits (see also Fig. 1).

No signal was detected in any other pointing at that position during the GCDE, nor during any other INTEGRAL observation of this sky region. The difference in the upper limits on the NRAO 530 count rate in the various pointings (Fig. 2, right) is due to the different location of NRAO 530 with respect to the ISGRI FOV: the farther the source from the FOV center, the more shallow its upper limit, due to the radially decreasing sensitivity of the ISGRI detector. Note that the upper limits obtained in the $\sim 2 \mathrm{~h}$ bracketing the NRAO 530 detection indicate that the flux change is highly significant. Therefore, the hypothesis that the source was constant and could only be detected when sufficiently close to the ISGRI FOV center is ruled out. We conclude that the detection of the rapid variation is genuine.

The total time on source is $1745 \mathrm{~s}$, that can be considered the minimum time scale of the event. It is worth mentioning that INTEGRAL performs two slews, one before and the other after the pointing, each $\approx 120 \mathrm{~s}$ long. It is not possible to detect the source during a slew in a coded-mask instrument (except for very bright cases, like GRB) and therefore it is not possible to say if NRAO 530 was flaring already during the slews. Taking into account also the marginal detection in the pointing following the central one the maximum time scale of the event is $\approx 1 \mathrm{~h}$.

We searched the SIMBAD catalog for Galactic hard X-ray sources within a $6^{\prime}$ radius (twice the ISGRI error radius for a source with this significance, see Gros et al. 2003) of the position of NRAO 530, that could possibly contaminate our ISGRI detection, but found none. Therefore, we attribute the flux detection entirely to NRAO 530.

The count rates of NRAO 530 in the $20-40 \mathrm{keV}$ energy band, already corrected for systematics, are: $2.7 \pm 0.5 \mathrm{c} / \mathrm{s}$ when using OSA 3, that correspond to $26 \pm 5 \mathrm{mCrab}^{3}$. When using OSA 5 , the rate is $3.2 \pm 0.6 \mathrm{c} / \mathrm{s}$, corresponding to $30 \pm$ 6 mCrab, consistent with the measurement with OSA 3. We take a weighted average as reference: $28 \pm 3$ mCrab. By adopting the standard spectrum of the Crab as reported by Toor \& Seward $(1974)^{4}$, the converted flux is $(2.1 \pm 0.2) \times$ $10^{-10} \mathrm{erg} \mathrm{cm}^{-2} \mathrm{~s}^{-1}$. No detection has been found in PICsIT, the high-energy layer of IBIS.

\section{Radio data}

We searched also for quasi-simultaneous data at other wavelengths and found only a radio observation at $2 \mathrm{~cm}$ (VLBA), made on 11 February 2004, a few days before the INTEGRAL detection, within the MOJAVE project $^{5}$ (Lister \& Homan 2005). The flux density was $4.1 \mathrm{Jy}$, with $3.1 \%$ linear polarization. The previous observation dates back to 9 October 2002, with a higher flux (5.7 Jy) but lower polarization $(0.5 \%)$. In 2005, lower fluxes and linear polarization have been observed. These data can be compared with the historical radio observations by Bower et al. (1997): as noted already by those authors there is no apparent correlation between the radio flux density and the optical activity in 1977-1978. NRAO 530 generally shows fluxes from a few up to more than $10 \mathrm{Jy}$, depending on the radio frequency. Therefore, the MOJAVE 2004 measurement of 4.1 Jy could indicate a low activity phase.

The polarization measurements reported by Bower et al. (1997) show an increase (up to about $8 \%$ at $8 \mathrm{GHz}$ ) during the two years preceding the optical outburst in 1977-1978. In our case, the MOJAVE measurement of a moderately high polarization value some days before the hard-X flare could be linked to the latter. We note that the present episode is a rapid event, not a long outburst like in 1977-1978, therefore the fact that only

\footnotetext{
3 We assumed $1 \mathrm{Crab}=105 \mathrm{c} / \mathrm{s}$ in the $20-40 \mathrm{keV}$ energy band of the IBIS/ISGRI detector.

4 Power Law model with $\Gamma=2.1$ and normalization

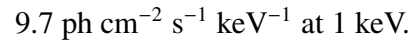

5 http://www.physics.purdue.edu/astro/MOJAVE/
} 


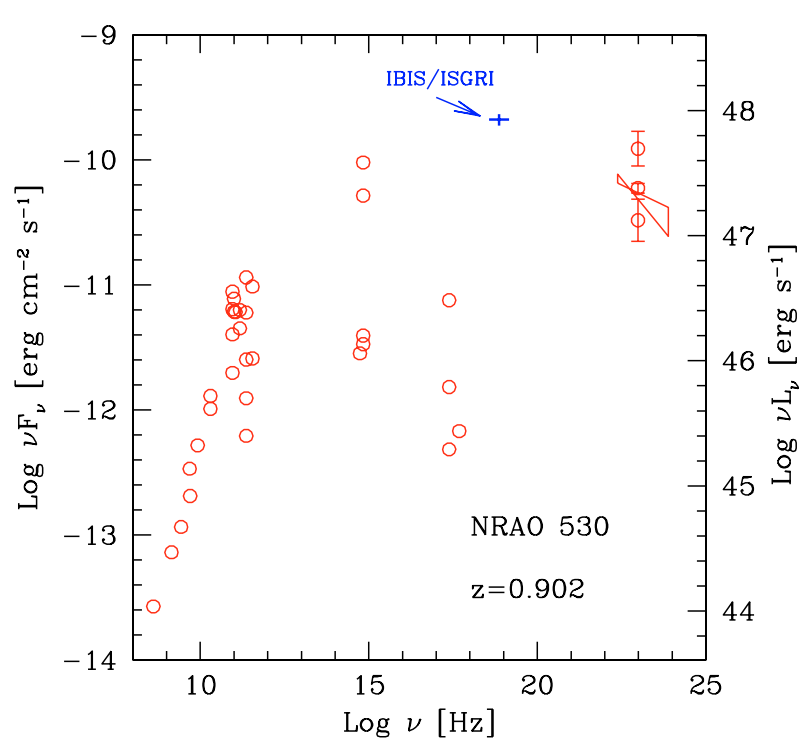

Fig. 3. Spectral energy distribution of NRAO 530 updated with the INTEGRAL data of the present work. Radio data from NED and Bower et al. (1997). Reference value of optical observations from Hewitt \& Burbidge (1993); historical maximum from Pollock et al. (1979), Webb et al. (1988). X-rays: ROSAT (Comastri et al. 1997), Einstein (Marscher \& Broderick 1981), HEAO-1 (Marscher \& Broderick 1981). $\gamma$-rays: CGRO/EGRET (Hartman et al. 1999). Blue cross: IBIS/ISGRI, present work.

moderate polarization was observed is not inconsistent with the fast flare in hard X-rays.

\section{Discussion}

Hard X-ray emission from the blazar NRAO 530 was detected serendipitously by INTEGRAL with IBIS/ISGRI during a long exposure of the Galactic Center. During a time interval lasting $\approx 2000 \mathrm{~s}$ the source flux in the $20-40 \mathrm{keV}$ range rose above the detection threshold by about a factor of $\sim 2$ and then faded again.

The spectral energy distribution of NRAO 530 constructed with historical data and with the present INTEGRAL flux is reported in Fig. 3. Interestingly, although very high, the IBIS/ISGRI point is consistent with the extrapolation to lower energies of the average spectrum observed in the EGRET energy range. The variability amplitude measured by EGRET was a factor 6 and rather erratic, still on timescales of weeks (Mukherjee et al. 1997). On the other hand, timescales as short as implied here would not have been accessible for EGRET due to the low counting rate. The hard X-ray flux measured during the flare for any reasonable spectral shape would imply a much larger X-ray flux than observed previously. Large variations have been observed from this object also in the optical (more than a factor 10 over timescales of years), thus overall the amplitude of variability is not unprecedented.

What is truly exceptional in this event is the short timescale, less than an hour in an object of extremely high luminosity. This may cast doubts on the association with NRAO 530. The event could then be attributed to some still unknown galactic source inside the $3^{\prime}$ radius circle of positional uncertainty in the ISGRI detection. In this case, only future satellites, like SIMBOL-X (Ferrando et al. 2005), will have the necessary spatial resolution at hard X-rays to confirm the association with NRAO 530. For the moment, with the present data, instrument performances, and catalogs, it is interesting to consider the possibility that this highly significant variation is associated with the blazar.

Rapid flares, with timescales of thousands of seconds in the X-rays, are often observed in BL Lac sources, especially in the TeV sources (e.g. Brinkmann et al. 2005), but they are rarely seen in blazars belonging to the quasar class, where typical timescales, even in hard X-ray- $\gamma$-ray band, are in the range of several hours-days (e.g. Wagner \& Witzel 1995). It is worth noting that FSRQ are generally faint in X-rays and therefore the above assertion could be biased in the past by the lack of high-energy astrophysics satellites with the necessary sensitivity. The present and the next generation of $\mathrm{X}$ - and $\gamma$-ray satellites should remove this bias.

The interpretation of such a rapid flare in the context of the standard synchrotron-inverse Compton models for blazars (e.g. Ghisellini et al. 1998) requires quite extreme conditions for the emitting source. A flare lasting less than $\Delta t=3600 \mathrm{~s}$ must be emitted by a region with a size $R<1.1 \times 10^{14} \delta \mathrm{cm}$, where $\delta$ is the Doppler factor. Adopting the value of $\delta=15$, suggested by VLBI observations (Bower \& Backer 1998), the size of the source is $R<1.6 \times 10^{15} \mathrm{~cm}$, an order of magnitude less than dimensions typically estimated for blazars $\left(10^{16}-10^{17} \mathrm{~cm}\right)$. In the case of a mass of the central supermassive black hole of $10^{8} M_{\odot}$, the above distance would correspond to only about 50 times the radius of the innermost stable orbit.

A direct alternative is to admit a large value of the Doppler factor, $\delta \sim 100$. Such large values of $\delta$ are sometimes invoked to explain the rapid intra day variability observed in the radio band (e.g. Wagner \& Witzel 1995). In this case, it would be possible to explain the short flare in terms of unsteadyness of the jet flow, due to a single non stationary shock (Hughes et al. 1985) possibly induced by a collision of two relativistic plasma shells in the jet (internal shock, Spada et al. 2001). The event discussed here could also result from an internal shock developping very close to the origin of the jet with less extreme values of $\delta$. The moderate polarization observed at radio wavelengths is consistent with these scenarios, since it arises from regions much further out in the jet.

Although the data available are few and sparse, it is possible that the present observation represents the "tip of an iceberg" leading the way to discover more short time scale events. We plan to continue our search of variability of blazars in the INTEGRAL field of view.

Acknowledgements. L.F. wishes to thank A. Domingo Garau for useful discussion about OMC data. This research has made use of the SIMBAD database, operated at CDS, Strasbourg, France, of the NASA/IPAC Extragalactic Database (NED) which is operated by the Jet Propulsion Laboratory, California Institute of Technology, under contract with the National Aeronautics and Space Administration, of data obtained from the High Energy Astrophysics Science Archive Research Center (HEASARC), provided by NASA's Goddard Space Flight Center, and of data from the Monitoring of Jets in AGN with VLBA Experiments (MOJAVE) Project. This work was partly 
supported by the European Community's Human Potential Programme under contract HPRN-CT-2002-00321 (ENIGMA).

Note added in proof. After acceptance of the manuscript, the Swift satellite performed two observations of NRAO530 (on 10 and 14 February 2006). During both observations, the X-Ray Telescope (XRT) detected only the blazar NRAO 530 within the ISGRI error circle of $3^{\prime}$. This result strengthens our conclusion that the exceptional flare detected by INTEGRAL is very likely due to NRAO 530.

\section{References}

Brinkmann, W., Papadakis, I. E., Raeth, C., Mimica, P., \& Haberl, F. 2005, A\&A, 443, 397

Bower, G. C., \& Backer, D. C. 1998, ApJ, 507, L117

Bower, G. C., Donald, C. B., Wright, M., et al. 1997, ApJ, 484, 118

Comastri, A., Fossati, G., Ghisellini, G., \& Molendi, S. 1997, ApJ, 480,534

Di Cocco, G., Caroli, E., Celesti, E., et al. 2003, A\&A, 411, L189

Ferrando, P., Goldwurm, A., Laurent, P., et al. 2005, Proc. SPIE 5900, 195

Foschini, L., Di Cocco, G., Malaguti, G., et al. 2005, ATel, 497

Ghisellini, G., Celotti, A., Fossati, G., Maraschi, L., \& Comastri, A. 1998, MNRAS, 301, 451

Goldwurm, A., David, P., Foschini, L., et al. 2003, A\&A, 411, L223

Gottesman, S. R., \& Fenimore, E. E. 1989, Appl. Opt., 28, 4344

Gros, A., Goldwurm, A., Cadolle-Bel, M., et al. 2003, A\&A, 411, L179

Hartman, R. C., Bertsch, D. L., Bloom, S. D., et al. 1999, ApJS, 123, 79

Hewitt, A., \& Burbidge, G. 1993, ApJS, 87, 451

Hughes, P. A., Aller, H. D., \& Aller, M. F. 1985, ApJ, 298, 301

Jorstad, S. V., Marscher, A. P., Mattox, J. R., et al. 2001, ApJ, 556, 738
Junkkarinen, V. 1984, PASP, 96, 539

Lamb, R. C., \& Macomb, D. J. 1997, ApJ, 488, 872

Lebrun, F., Leray, J. P., Lavocat, P., et al. 2003, A\&A, 411, L141

Lister, M. L., \& Homan, D. C. 2005, AJ, 130, 1389

Lund, L., Budtz-Jørgensen, C., Westergaard, N. J., et al. 2003, A\&A, 411, L231

Marscher, A. P., \& Broderick, J. J. 1981, ApJ, 249, 406

Marscher, A. P., Marshall, F. E., Mushotzky, R. F., et al. 1979, ApJ, 233, 498

Mas-Hesse, J. M., Giménez, A., Culhane, J. L., et al. 2003, A\&A, 411, L261

Monet, D. G., Levine, S. E., Casian, B., et al. 2003, AJ, 125, 984

Mukherjee, R., Bertch, D. L., Bloom, S. D., et al. 1997, ApJ, 490, 116

Pian, E., Malaguti, G., Maraschi, L., Ghisellini, G., \& Palumbo, G. G. C. 1999, ApL\&C, 39, 137

Pian, E., Foschini, L., Beckmann, V., et al. 2005, A\&A, 429, 427

Pian, E., Foschini, L., Beckmann, V., et al. 2006, A\&A, 449, L21

Pollock, J. T., Pica, A. J., Smith, A. G., et al. 1979, AJ, 84, 1658

Spada, M., Ghisellini, G., Lazzati, D., \& Celotti, A. 2001, MNRAS, 325,1559

Toor, A., \& Seward, F. D. 1974, ApJ, 79, 995

Ubertini, P., Lebrun, F., Di Cocco, G., et al. 2003, A\&A, 411, L131

Ulrich, M.-H., Maraschi, L., \& Urry, C. M. 1997, ARA\&A, 35, 445

Urry, C. M., \& Padovani, P. 1995, PASP, 107, 803

Vedrenne, G., Roques, J.-P., Schönfelder, V., et al. 2003, A\&A, 411, L63

Veron-Cetty, M. P., \& Veron, P. 2000, A\&AR, 10, 81

Wagner, S. J., \& Witzel, A. 1995, ARA\&A, 33, 163

Webb, J. R., Smith, A. G., Leacock, R. J., et al. 1988, AJ, 95, 374

Westergaard, N. J., Oxborrow, C. A., Chevenez, J., et al. 2005, JEM-X Science Analysis: Scientific Validation Report v 5.0, Danish National Space Center

Winkler, C., Courvoisier, T. J.-L., Di Cocco, G., et al. 2003, A\&A, 411, L1 